bed for eight months. The present trouble was attributed to lifting a heavy box. She was treated in Africa for hipjoint disease. On examination, the right hip-joint was nomal, and the thigh was slightly flexed on the abdomen. There was nothing abnormal to be noticed front or backno tumour or thickening in the pelvis to be made out. The patient complained of pain on walking or sitting on the right tuber ischil, pain on coughing, pain during micturition, radiating down to the pubes of the right side and down the right thigh; pain also on deep pressure in the right iliac fossa; pain on pressing the ilia together; pain also well marked on pressing immediately over the centre of the joint behind; occasional pain down the back of the right thigh and the right knee-joint. On walking, the patient stepped cautionsly, inclining the body to the right side and auvancing the right leg in front of the left. The toe was not everted. From these symptoms I had little doubt that disease existed in the sacro-iliac joint

Sir James Paget kindly saw the case with me on the following Sunday and confirmed my diagnosis. A double Thomas' splint was ordered and tonics prescribed. The patient would not submit to counter-irritation in any form, having, as she said, been " nearly killed with blisters in South Africa." After the application of the splint matters did not mend. The patient suffered from almost constant pain, her nights were sleepless, and in fact she was running down very fast. Some local heat was apparent over the jcint, but no general rise of temperature was at any time ayparent, although the thermometer was used regularly throughout the treatment. With a view to surgical interference, I removed the patient from her apartments to St. Thomas's Home. Here the case was agrain seen by Sir James Paget, in conjunction with Dr. Edmunds. I proposed to trephine the joint from the outside, and my proposal was not dissented trom, al though not recommended by niy colleagues. With the assistance of Dr. Edmunds and, in the presence of Mr. Makins and others, I proceeded to open the joint with a medium-sized trephine. When on the table a distinct swelling was noticeable over the joint about as large as the palm of one's hand. Examined per vaginam and rectum the pelvis was free from disease. A curved incision, eight inches in length, parallel with, and an inch below, the posterior third of the crest of the ilium, and descending vertically over the joint, exposed the bone sufficiently. The bone was next denuded with the elevator, and now was seen to be distinctly swollen and inflamed. A line being drawn from the anterior superior spinous process to the posterior superior, two inches were measured from this posteriorly. The pin of the trephine was placed on the line, so that the edge of the circle should be on the two-inch limb. The bone appeared softer than natural, the srephine rapidly penetrating to the joint. On removing the button of bone with the elevator a bed of soft Heshy vranulation tissue was reached, fully one-third of an inch in thickness. The opposing surfaces of the joint were quite lenuded of cartilage, and the bone on the sacral aspect was eroded, hard, and of a distinct yellow abnormal colour. The whole of the granulation tissue and dead bone, as far as could be reachel, was removed with the gouge and mallet, the joint swabbed with chloride of zine (forty grains to the ounce) and subsequently well dusted with iodoform. A large drainage-tube was inserted to the bottom of the opening, and the wound closed and dressed after the usual fashion. I need not weary my readers with the details of the case. Suffice it to say that all pain ceased immediately after the operation. The wound healed throughout by first intention, and at no time was a drop of pus ever present. The patient made an uninterrupted good recovery, and after a subsequent rest in the splint for six months left the home for the seaside, and bas now returned to South Africa.

in conclusion, I would recommend that all cases of sacroiliac disease where suppuration is threatening, or where pain is severe, be treated in a like manner.

New Carendish-street, $\mathbf{W}$.

Abervithian Sochety OF ST. Bartholomew's Hosprtal.--MIr. Oliver Pemberton, Professor of Surgery in Queen's Cullege, Birmingham, on the loth inst. delivered the introductory address on the occasion of the opening night of the session of this Society to an assembly of the staff, students, and nurses, the subject being on "The Progress of Surgery-a retrospect of forty years, and as to the measure of aid the School of St. Bartholomew afforded, both previously to and during that period."
THE

\section{TREATMENT OF PURULENT OPHTHALMLA AND DACRYOCYSTITIS BY ANTISEPTIC IRRIGATION.}

A NEW CANNULA HOR IRRIGATING THE LACRYMAL DUCT. BY HUGH E. JONES,

ASSISTANT SURGRON, LIVERPOOL EIE AND LAR INFIRMARY.

THE object of this short paper is to show what success has in practice followed the treatment advocated by $\mathrm{Mr}$. Edgar Browne for purulent ophthalmia and dacryocystitis. For the convenience of readers I have described the methods employed, and for those who may wish to try the treat. ment, the modifications which experience showed to be advisable. I am indebted to Mr. Richard Williams for permission to add two of his cases of gonorrhoeal ophthalmia to those of Mr. Edgar Browne.

In his paper on "Some Points in the Treatment of Purulent Ophthalmia" 1 Mr. Edgar Browne said that the indications for the treatment of that disease were (1) to wash away the infective material as thoroughly and as early as possible; (2) to render the conjunctival epithelium and secretions as nearly as may be aseptic. He then described his own method of following out these indications by prolonged irrigation of the whole of the conjunctiva with a $\frac{1}{3}$ per cent. solution of trichlor-phenolate of magnesium. The apparatus used consisted of an ordinary irrigating reservoir and tube and a lid retractor made of fine silver tubing perforated with six holes in the portion which entered the conjunctival sac. No cases of gonorrhoeal ophthalmia in which this treatment had been employed were mentioned in that paper, though the results obtained in urethral gonorrhoea were given.

\section{Chases of Purulent Ophthalmia.}

CASE 1.—J. G-_-, a man aged forty-nine, was admitted to hospital on March 12th, 1886, suffering from gonorrhœa and gonorrhoeal ophthalmia complicated by ulceration of the cornea. The lids were painted once with the nitrate of silver lotion (ten grains to the ounce). The conjunctiva was irrigated daily with trichlor-phenol solution. On March 17th the ulcer was healing. On the 22nd the discharge had quite stopped. The patient left well on the 30 th.

CASE 2.-J. T-, a man aged thirty, was admitted to hospital on July 27th, 1886, suffering from gonorrbœeal ophthalmia, complicated by a perforating ulcer of the cornea. The conjunctiva was irrigated three times a day with trichlor-phenol solution. By Aug. 4th the cornea was healing. There has been no extension of the ulcer since the patient's admission. On Aug. 13th the discharge had ceased. Cornea healed.

CASE 3.-M. P-, a woman aged thirty-eight, was admitted to hospital on Aug. 30th, 1887, suffering from gonorrhoeal ophthalmia and deep marginal ulcer of the cornea. The lids were painted, on admission, with the nitrate of silver lotion (twenty grains). Afterwards the treatment consisted simply of irrigation with Panas' solution of biniodide of mercury, and the instillation of eserine. The ulcer began to heal on the day after admission. The patient was discharged on Oct. 18th. The ulcer had healed perfectly without perforation, and there was no permanent impairment of the vision. The long stay in hospital is accounted for by the patient's destitute condition and the depth of the corneal ulcer.

CASE 4.-M. C-, a woman aged twenty-three, was admitted to hospital on Dec. 3rd, 1887, suffering from gonorrhocal ophthalmia and deep inarginal ulcer in the right eye, syphilitic iritis of the left, and well-marked syphilide on face and ulceration of tongue. The right eye was irrigated three times a day with Panas' solution, and the cornea dusted with powdered iodoform; the left eye was atropised. The ulcers of the tongue were touched with ac. hydrarg. nitratis and oleate of mercury ointment (10 per cent.) rubbed into the axillæ. The corneal ulcer began to heal the second day. On Jan. 20th the ulcer was quite healed. No discharge. Vision equals $\frac{20}{5}$; Jaeger 1 , each eye. Remarks. - With regard to the antiseptic solutions used, 
we found that Panas' solution of biniodide of mercury caused much less smarting than the trichlor-phenol (even when the latter was used as a $\frac{1}{2}$ per cent. solution), and that though the treatment occupied a longer time, the final result was quite as good. In Case I Mr. Edgar Browne's irrigating lid retractor was used; in the other three I used an ordinary Eustachian catheter bent to a suitable angle, and found that, when passed well up into the conjunctival sac and moved slowly from one end to the other, it acted as well, or nearly as well, as the more elaborate special instrument. ${ }^{2}$ In all the cases ulceration of the cornea had set in before admission to hospital, and in all healing began within a few hours of the first irrigation. The discharge was always much less on the second day, and ceased entirely within a fortnight, so that the patients could be transferred to the general wards. In all the cases $I$ have seen treated by the older methods (nitrate of silver, iodoform, \&c.) ulcers have gone on spreading until the discharge had nearly stopped, causing very severe permanent mischief to the cornea, and in some cases total loss. Irrigation was also found very useful in many cases of strumous and membranous ophthalmia, and in some of sloughing ulcer of the cornea with chronic conjunctivitis. In ophthalnia neonatorum, though shortening the duration of the disease very much, it was too troublesome and painful to be made the routine practice.

CASE 1.-A. B-'s (aged two years and a half) eyes became inflamed on June 30 th after an attack of diarrhœa. No exanthem. The patient was admitted to hospital on July 5th, suffering from severe membranous conjunctivitis and keratitis. The treatment consisted of irrigation with Panas' solution, iodoform ointment, and ott. atrop. (two grains to the ounce). On July (6th there was ulcer of the cornea. On the 8th the swelling of the lids had subsirled, the membrane had disappeared from the conjunctiva, and the ulcer was healing. The patient was discharged well on July 13th.

CASE 2.-K. G-—, aged two weeks, suffering from ophthalmia neonatorum, was seen on June 25 th as an outpatient. The conjunctiva were irrigated with trichlorphenol. The irrigation to be repeated daily. On June 29th the discharge had quite stopped. The patient was kept under observation until July 20 th ; but there was no recurrence of the discharge, though the treatment was limited to four irrigations.

\section{DACRYocystitis.}

In $1886 \mathrm{Mr}$. Edgar Browne, ${ }^{3}$ recognising the important part played by pyogenic cocci-as shown by the researches of Saittler and Widmark-in diseases of the lacrymal sac and duct, applied the principle of irrigation to the treatment of lacrymal disease. As house surgeon it fell to my lot to carry out the details of the treatment. The object to be attained was practically the same as in gonorrhceal ophthalmia-viz., to wash away the discharge and to render the mucous membrane and its secretion aseptic without damaging the epithelium or agravating the existing inflammation. In the first few cases an ordinary No. 1 gum-elastic catheter, to which the irrigator tube was attached, was passed down the lacrymal duct and trichlor-phenol solution (about $\frac{1}{2}$ per cent.) used; but this was never a very satisfactory arrangement, as it was difficult to pass the catheter, and the solution caused very severe smarting. In all the cases recorded here the following was the method adopted. 1. The lower canaliculus was slit up sufficiently to admit a moderatesized probe (except in some cases of lacrymal abscess, where the external opening rendered this unnecessary). 2. Weber's stilette and cannula (made by Matthieu, and figured in Juler's "Ophthalmic Science and Practice") were then introduced into the sac, the stilette withdrawn, the irrigator tube attached to the cannula, and about half a pint of Panas' solution run through. 3. Next day, supposing the sac not to be too acutely inflamed, a probe was passed, and afterwards the whole tract irrigated, the cannula being passed down the duct. In chronic cases, where the discharge was too thick and viscid to yield to ordinary means, about a pint of a ten-grain solution of bicarbonate of soda was used before the Panas' solution. 4. When a stricture existed a larger cannula was used every day until the stricture was sufficiently dilated. 5. During the process of irrigation it is necessary for the patient to hold his mouth wide open, for obvious reasons.

2 A hollow irrigating speculum has been invented by $\mathrm{Wr}$. Treacher Collins (vide THE LANCET, vol. i., 1885).
The following objections to the instrument will probably occur to every ophthalmic surgeon: l. If at all strongly curved, the withdrawal of a stiff, closely fitting stilette must be a difficult and jerky process. 2. If the cannula is introduced up to the hilt, the fluid irrigates not tho lacrymal sac or duct, but the nasal mucous membrane. 3. If the cannula be withdrawn ever so slightly, it cannot, owing to its open end, be replaced without reintroducing the stilette. To avoid these difficulties, I got Messrs. Weiss and Son to make a cannula closed and rounded at

Fig. 1.

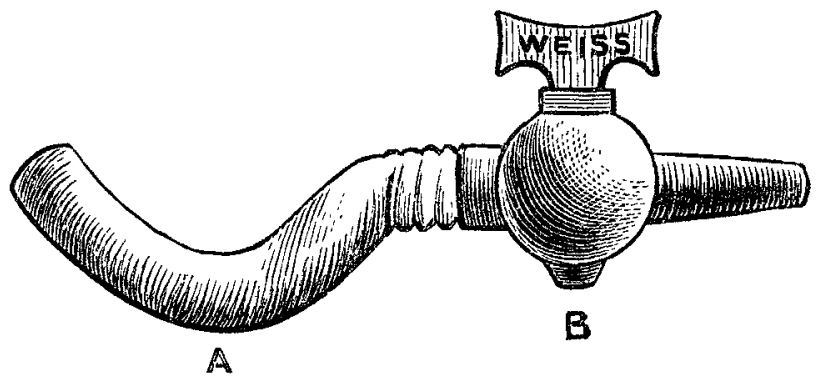

A, Rubber tubing from reservoir. B, Stopcock.

FIr. 2.

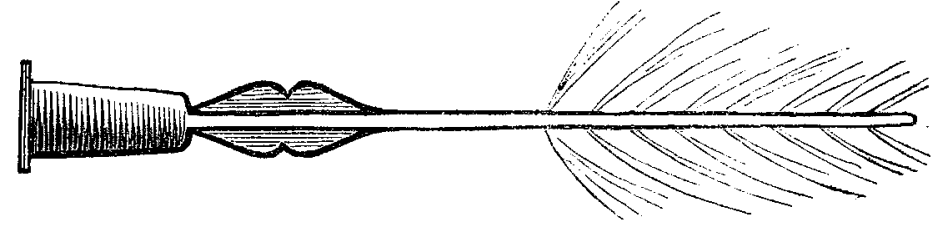

Cannula (small bore).

the distal end, and pierced along the sides by small holes for about one inch of its length. The proximal end was made to fit the tapering nozzle of a small stopcock. No stilette is required, for this cannula can be withdrawn and reintroduced to any extent with perfect ease and without detaching the stopcock; it, moreover, irrigates the whole of the sac, nasal duct, and part of the nasal fosse at the same time. I used this cannula daily for four months. It serves its purpose admirably, and is kept clean quite easily.

CASE 1.-R.M M-, aged thirty-seven. Seven months ago the patient had muco-purulent discharge from the lacrymal duct for three months, culminating in an abscess of the sac. This was followed by mucocele (four months), on account of which he came to the infirmary on Nov. 5th, 1886. The lower canaliculus was incised and a probe passed. On Nov, 6th, 7th, 8th, 12th, 14th, and 16th the duct was irrigated with Panas' solution. On the last-mentioned date the patient was quite well, there being no discharge or epiphora. In reply to questions, he wrote on Feb. 20th, 1889 , saying that there was a slight watering of the eye six months after his last attendance, but that there had been no recurrence of the mucocele or aluscess. The duration of treatment was eleven days ; the number of irrigations six.

CASE 2.-E. A-_-, aged forty, was seen as an out-patient on July 2nd, 1886, with lacrymal obstruction and blennorrhœa. On July 2nd the lower canaliculus was slit. Probes were passed and boracic acid collyrium was used regularly until Oct. 25th, 1886 -i. e., 114 days. From Uet. 25 th and every day until Nov. 4th irrigation was practised. On Nov. 4th the mucons membrane was perfectly smooth and there was no discharge or epiphora. The patient remained under observation until Jan. 24th, 1888, without further treatment (except for mixed astigmatism and squint), during which time no recurrence of epiphora took place. Duration of treatment by irrigation eleven days.

CASE 3.-D. B-, aged twenty-six, was admitted to hospital on Jan. 25th, 1887, suffering from an old lacrymal abscess with recent recurrence. The abscess was incised. On Jan. 26th and 27 th the abscess was irrigated, and the patient went home. On Feb. lst fresh inflammation and discharge from the sac supervened. On the 4th the swelling subsided. As the patient lived at a distance, it was not possible to irrigate the duct oftener than once a week, and sometimes a fortnight elapsed without any treatment being applied. On March 4th rough bone was felt in the upper part of the duct. The patient ceased attending on April 19th. On this date he was quite well; no swelling or discharge, and duct smooth. During the whole of 1887 
and 1888 the patient was seen at intervals of three or four months. Sometimes a probe was passed to test the patency of the duct, but no sign of stricture was ever discovered. Duration of treatment eleven weeks ; number of irrigations about twelve.

CASE 4.-E. Q - aged thirty-three. Lacrymal obstruction and blennorrboe off and on for two years. Threatening abscess. On Aug. 5th, 1887, the canaliculus was slit and probes were passed. The blennorrhoea continued undiminished until Sept.9th, when irrigation was begun and carried out daily until Sept. 20 th, when the patient was quite well. In answer to questions, the patient wrote on Feb. 20th, 1889, that there had been no discharge from, or watering of, the eye since her last attendance in September, 1887. Duration of treatment by irrigation eleven days.

CASE 5.-W. S-, aged twenty-eight. Epiphora for three years, followed by mucocele. The canaliculus was slit and irrigation performed on Oct. 19th, 1886. The irrigation was repeated on Oct. $22 \mathrm{nd}, 24 \mathrm{th}$, and $26 \mathrm{th}$. On Nov, 19th the patient was quite well. Duct irrigated. Jan, 18th, 1887 (i.e, after trvo months), there was some thickening over the lacrymal sac, but no epiphora or discharge. On Feb. Woth, 1889 (i.e., two vears and three nonths after cessation of treatment), the patient wrote to say that he was quite well. Duration of active treatment seven days; number of irrigations five.

CASE 6.-J. H-_, aged sixty. Lacrymal abscess; ozena. On May 20 th the lower canaliculus was slit, and probes passed daily, without any improvement, until June 17 th. The duct was then irrigated with bicarbonate of soda lotion, followed by Panas' solution. This was done once a week until $\mathrm{July} 22 \mathrm{nd}$, when the discharge had entirely stopped. I was unable to trace this patient, so that I cannot say how long he remained well.

CASE 7.-A. M-, aged thirteen. Abscess of lacrymal sac. The abscess was incised, and irrigated daily for nine days. Twc years later the patient was perfectly well. No recurrence of abscess or epiphora.

Remar7s. - It may be asked whether this treatment has any lasting effect upon the stricture which, in many cases, has caused the mucocele or abscess. Cases 2 and 4 seem to me to show that it has, and the large number of cases treated during two years and a half have left a firm impression on my mind that the good effect is more permanent than in cases treated simply by the knife and probe, and that the formation of a stricture is more frequently pre. vented than by the ordinary methods of treatment, including the injection of astringents. Irrigation gains additional value from the fact that by it the nasal mucous membrane is treated simultaneously with that of the nasal duct and lacrymal sac, which must be of considerable importance in cases arising from or complicated by nasal catarrh or ozcena. find that I have inadvertently included one or two cases which were mentioned by $M_{r}$. Edgar Browne in his paper in the Practitioner, but as they were then quite recent, and as I have since been able to trace them up to 1889 , I have allowed them to remain. Many more cases might be given, but I think the above are sufficient to prove that the treatment advocated yields good results easily and rapidly.

P.S.-Since the above paper was written $\mathrm{Mr}$. Treacher Collins has told me that during his house surgeoncy at Moorfields he used a cannula similar to the one described above, with a svringe. I am inclined to lay some stress upon the advantage of using an irrigating reservoir instead of a syringe.

Liverpool.

SAlFoRd Royal Hospital. - The annual meeting of the subscribers to this institution was held on the 10th inst. in the board-room of the hospital, there being a rood attendance. The report showed that during the past year 15,314 patients had been treated, against 14,152 in the previous year and 13,101 in 1887 . The income had been $£ 4826$ and the expenditure $£ 5955$, leaving a deficit of $£ 1129$. 'The board had made an important modification in the way of admitting out and home patients in districts where there were few or no subscribers to the charity, and where there was consequently considerable difficulty in obtaining recom mends. They had provided 1 rinted forms, which when signed by two hruseholders certifying that the individual was a proper object of charity, entitled the applicant to medical assistance The report was adopted.

\section{Clinital alltes:}

\section{MEDICAL, SURGICAL, OBSTETRICAL, AND THERAPEUTICAL.}

\section{ON ANTIPYRIN.}

By Adolphus J. Richardson, M.A., M.D. Cantab., ASSISTANT PHYSICIAN TO THE SUSSEX COUNTYY HOSPITAL.

The note on Antipyrin in THE LANCET of Aug. 17th, 1889, by $\mathrm{Mr}$. Wingrave, induces me to give some details of its administration in a series of ninety-six cases, of which I have notes more or less complete. In only four of these has any bad effect been attributed to the drug. In a pregnant woman suffering from left-sided hemicrania, twenty minutes after she had taken ten grains an itching "white raised rash" appeared, diagnosed by a chemist as due to mussel poisoning. She had, however, taken nothing to which I could attribute it save the antipyrin. A patient with tabes dorsalis, after taking twenty grains fainted away, and is said to have lain insensible on the floor. One patient vomited after a dose of twenty grains, and one after a dose of ten grains; both, however, took it during a paroxysm of migraine, so it is very probable that the drug was not wholly to blame. In only the two above-mentioned cases was a larger initial dose than ten grains employed; I shall not repeat it. Thirty-eight of the cases were typical migraine; in nearly all I ordered ten-grain doses, to be repeated in an hour if the pain did not abate. In thirty-two cases the improvement was definite, in two doubtful, and four were unbenefited. In the successful cases, as a rule, not only were the paroxysms relieved, but their frequency was diminished. In three after a time the power of the drug seemed to fail, but an increase of the dose to fifteen grains produced the initial good result. In some cases, although the paroxysms were always relieved, their frequency was in no way affected. In none was the frequency increased. In two of the cases peptonuria was constant; both were relieved as to the migraine. The condition of the urine was observed in intervals as well as in paroxysms. Of the four cases in which the drug failed, one had, I believe, mitral obstruction, through which the migraine might have been aggravated; one vomited the antipyrin, probably before any therapeutic effects could have been obtained; the other two cases were both definitely hemicranias, and I have sometimes been tempted to think that the more definitely the migraine conformed to this type the less good was to be expected from antipyrin. In sixteen cases of head pain, some of which I could not explain at all, and none of which were markedly typical, the drug relieved in eight. A very different proportion to that on migraine, showing that the administration of the drug in a purely symptomatic manner meets with no undeserved success.

To detail the cases in which it failed:- - Headache in anæmia, with possible gastric ulcer. 2. Violent vertical headache, possibly syphilitic. 3 Headache, associated with astigmatism (unrelieved by glasses) and gastralgia; improved under arsenic. 4. Vertical headache in a phthisical patient. 5. Rheumatism of scalp. 6. Continuous headache "all her life." 7. Temporal headache, possibly middleear disease. 8. Temporal headache.

In three cases of anremic headache in chlorotic patients, contrary to my expectation, the drug relieved. These were not cases of migraine in chlorotic girls, but were headaches which were worse in the morning, unaccompanied by sick. ness or visual phenomena, and which got better as the day wore on, or on lying down. In four cases of tabes dorsalie, with lightning pains, my administration of the drug met with the most favourable results. All now invariably keep it ready for immediate use. One of the patients suffered from the most distressing nocturnal rectal tenesmus; on this the drug, although tried repeatedly, had no effect, but his lightning pains, of the most severe description, were at once relieved. Four times I have ordered ten grains to be taken at the onset of an attack of asthma nervosa, in all wiuhout any relief of the spasm. In three cases of facial neuralgia (in none of the patients was there any dental caries), one was much relieved and two but slightly; and it 